\title{
The development of a universal approach to testing of walkway slip resistance in the U.S.
}

\author{
James Flynn, PE ${ }^{\mathrm{a}, *}$, Steven Di Pilla, ARM, $\mathrm{CXLT}^{\mathrm{b}}$ and Keith Vidal PE, CXLT ${ }^{\mathrm{c}}$ \\ ${ }^{a}$ J2 Engineering, 5234 E. Pine Avenue, Fresno, CA 93727 \\ ${ }^{\mathrm{b}}$ ESIS Health, Safety \& Environmental, Philadelphia, PA \\ ${ }^{\mathrm{c}}$ Vidal Engineering St Louis, MO
}

\begin{abstract}
The measurement of walkway slip resistance is required to assess the risk of slipping. Unlike other countries, the U.S. has not decided upon a single approach to the measurement of slip resistance. Several types of tribometers are available to measure slip resistance however, the measured value of the slip resistance of a given surface has been found to be tribometer specific. The introduction of ASTM International Standard F2508 Standard Practice for Validation and Calibration of Walkway Tribometers Using Reference Surfaces, has produced a method which allows validation of each type of tribometer and the values generated during testing.
\end{abstract}

Keywords: Tribometer, Slip, Walkway safety, Slip resistance

\section{Introduction}

The measurement of walkway slip resistance is necessary to assess the risk of slipping. In the U.S., there has been considerable discussion, debate, and controversy regarding the selection of a definitive test method and apparatus for determining walkway slip resistance. Unlike several other countries, there has never been a single approach to such measurement and many different types of tribometers are used. No tribometer has been accepted which is able to measure slip resistance such that the measurements can be definitively related to human ambulation. The use of several different types of tribometers is problematic in that, when metering a walkway surface, the measurements generated have been found to vary with the tribometer used. This is not unexpected as the measurements are a function of the material being tested, the test foot material and the specific tribometer ${ }^{[2-9]}$.

Over the past 10 years, ASTM International, a consensus standards making organization, has worked to develop a slip resistance test standard for walkway surfaces that can be used to validate the use of any tribometer. In 2011, ASTM International published F-2508 Standard Practice for Validation and Calibration of Walkway Tribometers Using Reference Surfaces ${ }^{[1]}$. This standard practice is accompanied by an adjunct consisting of four tiles. The tiles are reference surfaces with surface slip resistance properties defined by human ambulation studies. Through the use of the F-2508 Standard Practice and the reference tiles, it can be determined whether or not a specific tribometer can produce valid measurements of the slip resistance of a walkway surface. At the same time, a method is provided by which the values of slip resistance generated from tribometrictesting of a walkway surface can be related to human ambulation $^{[5,9]}$.

\section{Methods}

With the acceptance of the use of several types of tribometers, it was necessary to develop a method for

\footnotetext{
${ }^{*}$ Corresponding author.

E-mail address: jflynn@j2eng.com Phone: 1 (559) 251-5600
} 
an objective evaluation of tribometer performance. To that end, reference surfaces were selected for tribometer testing. Four types of tiles were selected for testing. The tiles were polished black granite, porcelain, ceramic and vinyl composition tile (VCT). To rank the tiles in order of slip resistance, testing was conducted at the University of Southern California Musculoskeletal Biomechanics Laboratory. Eighty subjects were recruited to walk across the tiles and the subjects were monitored using an 8-camera Vicon motion analysis system. The tiles were wetted prior to each trial. All slips were recorded and using the number of slips per tile, the tiles were ranked in order of slip resistance. The polished black granite was most slippery and was followed in decreasing order by porcelain, VCT and ceramic ${ }^{[9]}$.

With the availability of the reference surfaces, ASTM International Committee F-13 Pedestrian Walkway Safety and Footwear, created ASTM International Standard Practice F-2508. The standard involves using the reference tiles to validate tribometer performance. In order for the performance of a tribometer to be considered valid, the tribometer must satisfy two criteria. It must be able to statistically differentiate between the surfaces of the four reference tiles. The tribometer must also be able to rank the slip resistance of the tiles in the same order as that generated by the human subject testing at the USC Musculoskeletal Biomechanics Laboratory.

If the tribometer can differentiate between the tiles and rank the surfaces in the correct order, the testing of the reference tiles can provide tribometer specific slip resistance values for the four different reference surfaces. The values for slip resistance generated during testing of the reference surfaces can be used as benchmarks for the testing of an unknown surface as the validity of the values can be traced back to the human subject testing. If a surface is measured and is found to have a slip resistance value of 0.4 , the value only has meaning with respect to the results obtained from testing the reference tiles. If, when testing the reference tiles, values from the polished black granite were close to 0.4 , then 0.4 would indicate a slippery surface. If however, testing the non-slip surface of the ceramic tile generated a value of 0.4 , a value of 0.4 would indicate a non-slippery surface. It is possible that two tribometers testing the same reference tiles will get very different values. The values obtained from the testing of the reference tiles and the interpretation of those values are tribometer specific $[5,9]$.

The F-2508 Standard Practice is intended for the use of the manufacturer of each type of tribometer as well as the end user. The manufacturer conducts Walkway Tribometer Validation Testing while the end user conducts Walkway Tribometer Calibration Testing.

When conducting the Walkway Tribometer Validation Testing, forty tests are conducted on each tile. Prior to testing, each tile is wetted. The tiles are tested in four orthogonal directions. The results of testing are used to calculate the mean, standard deviation, standard error of the mean and the $95^{\text {th }}$ percentile confidence interval. The results of the analysis should show that the tribometer was able to produce significantly different results when measuring the slip resistance of the each of the surfaces. Significant difference is determined through the use of paired $t$ tests $(p<0.05)$ for all adjacently ranked reference surfaces.

When the end user is conducting Tribometer Calibration Testing, sixteen tests are to be conducted on each reference surface. The results of the testing are used to calculate the mean. Calibration requires the mean to be inside the $95^{\text {th }}$ percentile confidence interval as reported by the tribometer manufacturer.

End user results are reported based upon a specific tribometer and test foot combination. Calibration is therefore required whenever a new test foot is employed. Calibration is also required on an annual basis or whenever the tribometer is repaired ${ }^{[1]}$.

\section{Discussion}

The human subject testing at the USC Musculoskeletal Biomechanics Laboratory and the resultant creation of the ASTM International standard F-2508 are a significant movement in the direction of validation of tribometers and the measurements of walkway slip resistance. It must be noted that the standard does not state that the validation will hold for all combinations of walkway surfaces and test foot materials.

The ASTM F-13 Committee continues to move forward in the area of tribometry. The Committee is currently working on a new standard and a new guide. The first is the Standard Practice for Certifying Walkway Tribometers while the second is the Standard Guide to Walkway Auditor Qualifications.

The Standard Practice for Certifying Walkway Tribometers requires a Walkway Tribometer Validation Report as defined in Standard F-2508. It also requires the manufacturer to conduct a precision study through the ASTM International Interlaborato- 
ry Study office (ILS). The precision study looks at both tribometer repeatability and reproducibility. The repeatability is determined through the use of a single tribometer and single operator. The tribometer is used to test the reference tiles and focuses on the repeatability of the measurements from each specific reference tile.

Reproducibility involves the use of a minimum of six tribometer/operator combinations. Each tribometer/operator measures the slip resistance on the same group of four reference tiles. The data is used to determine the expected data spread between tribometer operators.

The precision study has no required minimum range for either repeatability or reproducibility. It simply provides a confidence level for those looking at data from different tribometers of the same type.

The Standard Guide to Walkway Auditor Qualifications is intended to provide an overview of the materials to be included in any program used to certify walkway auditors. The guide is designed to define a minimum competence level for those involved in walkway auditing.

\section{Summary}

ASTM International has created Standard F-2508 and a program within Committee F-13 which can be used to validate the use of a specific tribometer or tribometer type. The standard further can provide meaning to the values of slip resistance measurements when using a validated tribometer and the associated reference tiles.

\section{References}

[1] ASTM F2508, 2011, "Standard practice for validation and calibration of walkway tribometers using reference surfaces," ASTM International, West Conshohocken, PA, 2011, DOI: 10.1520/F2508-11.

[2] Gronqvist R, Hirvonen M, Tohv A. Evaluation of three portable floor slipperiness testers. Int J Ind Ergon 1999;25:85-95.

[3] Chang WR, Matz S. The slip resistance of common footwear materials measured with two slipmeters. Appl Ergon 2001; 32(6):548-58.

[4] Kulakowski BT, Buczek FL, Cavanagh PR, Pradhan P. Evaluation of performance of three slip resistance testers. J Test Eval 1989;17(4):234-40.

[5] Powers CM, Brault JR, Stefaou MA, Tsai YJ, Flynn J, Siegmund GP Assessment of walkway tribometer readings in evaluating slip resistance: a gait-based approach. J Forensic Sci 2007;52(2):400-5.
[6] Marpet MI, Comparison of walway safety tribometers. J Test Eval 1996;25(9):245-54.

[7] Marpet MI. Comparison of wakway safety tribometers: part two. J Test Eval 1997;25(1):115-26.

[8] Powers CM, Kulig K, Flynn, J, Brault JR. Repeatability and bias of two walkway safety tribometers. J Test Eval 1999;27(6):368-74.

[9] Powers CM, Blanchette MG, Brault JR, Flynn J, Siegmund GP. Validation of walkway tribometers: establishing a reference standard. J Forensic Sci. 2010;55(2):366-70.

[10] Medoff H, Fleisher DH, Di Pilla S. Comparison of slip resistance measurements between two tribometers using smooth and grooved Neolite test-liner test feet. In: Marpet MI, Sapienza MA. Editors. Metrology of pedestrian locomotion and slip resistance. ASTM STP 1424. West Conshohocken, PA: ASTM, 2002:67-72.

[11] Cham R, Redfern MS. Heel contact dynamics during slip events on level and inclined surfaces. Saf Sci 2002;40(7):55976.

[12]Powers CM, Burnfield JM, Lim P. Brault JR, Flynn JE. Utilized coefficient during walking: static estimates exceed measured values. J Forensic Sci 2002;47(6):1-6 